\title{
Resiliencia de redes de agua sectorizadas
}

\section{Resilience of district metered areas of water distribution networks}

Presentación: 6 y 7 de octubre de 2020

\section{Doctorando:}

Jezabel D. Bianchotti

Laboratorio de Métodos y Simulaciones computacionales, Facultad Regional Rafaela, Universidad Tecnológica Nacional - Argentina jezabelbianchotti@gmail.com

\section{Director/es:}

\section{Gabriel D. Puccini}

Co-director/es:

\section{Omar Chiotti}

\section{Resumen}

En este trabajo se propone una metodología para la sectorización óptima de redes de distribución de agua impulsadas por bombas y, además, se analiza el rendimiento de las soluciones obtenidas. El algoritmo de sectorización consta de dos etapas. La primera se plantea como un problema de optimización de múltiples objetivos y consiste en identificar una estructura de comunidades que privilegian la conectividad interna sobre la externa. La segunda etapa consiste en determinar la ubicación óptima de los dispositivos a instalar (válvulas y caudalímetros) en los cortes virtuales establecidos en las soluciones de la primera etapa. Para analizar el rendimiento de las soluciones se evalúa la variación del índice de resiliencia frente a incrementos en las demandas de un número fijo de nodos para cada una de las configuraciones de sectorización obtenidas.

Palabras clave: Redes de agua potable, sectorización, confiabilidad, resiliencia

\begin{abstract}
This paper presents a methodology for the optimal partitioning of water distribution networks including a pump system and, in addition, it analyzes the performance of each solution. The partitioning algorithm is based on a twostage approach. The first one is formulated as a multi-objective optimization problem and consists on identifying the optimal network partition, namely those communities with nodes interconnected closely, while the connection between communities is sparse. The second one consists on determining the optimal location of isolation valves and flow meters by using the results established in the first stage. To analyze the performance of the solutions, the resilience index is evaluated by considering the demand increments for a fixed number of consumers.
\end{abstract}

Keywords: Water distribution networks, sectorization, reliability, resilience

\section{Introducción}

Las redes de distribución de agua potable están compuestas por un grupo de diversos elementos, principalmente tuberías que, trabajando a presión y ubicadas en el espacio público, van entretejiendo las ciudades para transportar agua de forma segura desde las fuentes hasta cada uno de los consumidores. La mayoría de estas redes han sido diseñadas y construidas en el siglo pasado, y en la actualidad suelen experimentar fugas y fallas debido al deterioro de los artefactos que la componen y a los incrementos en las demandas producidas por el crecimiento urbano.

La sectorización de las redes de agua potable es una técnica dirigida a mejorar la gestión operativa del sistema hidráulico, que incluye la gestión de fugas y presión, monitoreo de la calidad del agua, facilitar las actividades de reparación y optimización de los sistemas de bombeo, entre otros. La idea central de esta técnica es establecer 
comunidades autónomas que pueden aislarse de la red mediante la instalación de válvulas de aislación y caudalímetros en tubos óptimamente seleccionados.

Tradicionalmente la sectorización de las redes se realizaba siguiendo sugerencias empíricas o por prueba y error, pero con el paso de los años se han propuesto métodos más sofisticados (Alvisi y Franchini, 2013; Di Nardo et al., 2014; Castro-Gama et al., 2016). Sin embargo, la dificultad adicional que se presenta a estas metodologías es que no existe una estrategia general para la segmentación de redes de agua debido a la falta de un índice que sea universalmente aceptado y que caracterice la estructura en comunidades o módulos de la red.

En la teoría de redes complejas, el índice de modularidad (Newman y Girvan, 2004) se propone como métrica para caracterizar la estructura en comunidades de una red. Sin embargo, el uso directo de este índice que ha sido definido sobre consideraciones netamente topológicas, no está totalmente aceptado en los sistemas hidráulicos debido a que estos son fuertemente dependientes de parámetros físicos. Para compensar este aspecto, se introduce el concepto de resiliencia, que fue desarrollado por Todini (2000) como una métrica para caracterizar la confiabilidad hidráulica del sistema y que puede interpretarse como el excedente de energía disponible en la red para el abastecimiento.

En este trabajo se propone una metodología de sectorización basado en dos etapas, cada una de ellas se plantea como un problema de optimización de dos objetivos que incorpora los conceptos de modularidad y resiliencia. Adicionalmente, se evalúa el rendimiento de las soluciones obtenidas bajo diferentes escenarios de demandas. La metodología se aplica usando una red de estudio académica.

\section{Desarrollo}

\section{Índice de Modularidad}

La topología de la red de agua puede describirse como un grado cuyos vértices representan los nodos y los enlaces representan los tubos. Así una red que contiene $N_{n}$ nodos se representa por una matriz de adyacencia $\boldsymbol{A}$ de tamaño $N_{n} \times N_{n}$, donde los elementos $A_{i j}=1$ si los vértices $i$ y $j$ están conectados, en caso contrario ese elemento será cero. La matriz de adyacencia permite definir el índice de modularidad (Newman y Girvan, 2004; Newman, 2006):

$$
Q=\frac{1}{2 m} \sum_{i j}\left(A_{i j}-\frac{k_{i} k_{j}}{2 n_{p}}\right) \delta\left(s_{i}, s_{j}\right)
$$

donde $m$ denota el número de enlaces (tubos) en la red, $k_{i}$ es el grado de vértices de $i$, y $\delta\left(s_{i}, s_{j}\right)$ es 1 si $i$ y $j$ están en el mismo grupo, y $O$ en caso contrario. Los valores cercanos a $Q=1$, indican la mejor estimación de la estructura en comunidades de la red. Nótese que esta medida de la división en módulos está basada solamente en propiedades de conectividad de la red.

\section{Índice de resiliencia}

La sectorización física de la red se consigue al introducir válvulas de aislación en tubos que delimitan comunidades, pero esta técnica puede reducir el rendimiento del sistema y con ello, disminuir su capacidad para sobrellevar fallas hidráulicas. El concepto de resiliencia fue introducido por Todini (2000), como medida de la confiabilidad hidráulica y habitualmente se usa para mejorar el rendimiento del sistema de agua bajo condiciones de falla, proporcionando mayor potencia en cada nodo que la mínima requerida. El índice de resiliencia se define como:

$I_{R}=1-\frac{P_{i n t}^{*}}{P_{m a ́ x}^{*}}$

donde $\mathrm{P}_{\text {int }}^{*}=\gamma\left(\sum_{k=1}^{N_{r}} Q_{k} H_{k}+\sum_{p=1}^{N_{b}} Q_{p} H_{p}-\sum_{k=1}^{N_{n}} q_{i}^{*} h_{i}\right)$ es la potencia disipada en la red para satisfacer la demanda total, y $P_{\text {máx }}^{*}=\gamma\left(\sum_{k=1}^{N_{r}} Q_{k} H_{k}+\sum_{p=1}^{N_{b}} Q_{p} H_{p}-\sum_{k=1}^{N_{n}} q_{i}^{*} h_{i}^{*}\right)$ es la máxima potencia disipada internamente para satisfacer las restricciones en términos de demanda $q_{i}^{*}$ y presión $h_{i}^{*}$ en el nodo $i$. Asimismo, $q_{i}$ y $h_{i}$ son las demandas y las presiones de cada nodo $i, N_{n}$ es el número de nodos, $Q_{k}$ y $H_{k}$ son la descarga y la presión, respectivamente, de cada 
reservorio $k$, siendo $N_{r}$ el número de reservorios, $Q_{p}$ y $H_{p}$ son la descarga y la presión aportadas por la bomba de agua, respectivamente, de cada bomba $p$, siendo $N_{b}$ el número de bombas, y $\gamma$ el peso específico del agua. Nótese que una red con un valor de resiliencia cercano a uno indica una red con buena capacidad para sobrellevar fallas hidráulicas.

\section{Formulación del problema}

El procedimiento de sectorización se plantea en dos etapas. La primera etapa consiste en identificar la estructura de comunidades que privilegian la conectividad dentro de cada módulo respecto a la conexión entre ellos. Las variables de diseño del problema de optimización están dadas por el conjunto de cortes virtuales $C_{j}$ que definen los módulos de la red. Esta etapa se plantea como un problema de optimización de dos objetivos:

$$
\begin{aligned}
& \operatorname{máx}(Q)=\operatorname{máx}\left\{\frac{1}{2 m} \sum_{i j}\left(A_{i j}-\frac{k_{i} k_{j}}{2 m}\right) \delta\left(s_{i}, s_{j}\right)\right\} \\
& \min \left(N_{C}\right)=\min \left\{\sum_{j}^{m} C_{j}\right\}
\end{aligned}
$$

donde $C_{j}=1$ si el j-esimo tubo conecta dos módulos, de otra manera $C_{j}=0$. Así, la función de costo que se busca minimizar es igual al número total de cortes virtuales $N_{C}$ que limitan los módulos. El problema de optimización formulado en la Ec. (3) proporciona un conjunto de soluciones que representan el compromiso entre el número de cortes y la capacidad de la red para dividirse en módulos. Es importante notar que no todos estos cortes, así obtenidos, podrán hacerse reales puesto que la segmentación fue realizada teniendo en cuenta solamente la topología de la red sin resolver el sistema hidráulico, por lo que, podrían generarse presiones deficientes o delimitarse módulos sin fuente para abastecer el sector.

A partir del conjunto de soluciones proporcionado por la primera optimización, se selecciona una solución particular para la segunda etapa. Con ella se busca determinar la ubicación óptima de los dispositivos a instalar: caudalímetros, en los enlaces donde los cortes no puedan materializarse y válvulas de aislación, donde los cortes puedan hacerse reales. Estas válvulas determinan la estructura de comunidades autónomas, y permiten que en circunstancias especiales, como cortes de servicio por reparación, o por presencia de contaminantes, se pueda aislar un sector sin afectar el funcionamiento del resto de la red.

La formulación del segundo problema se plantea como un problema de optimización de dos objetivos:

$\operatorname{máx}\left(I_{R}\right)=\operatorname{máx}\left(1-\frac{P_{i n t}^{*}}{P_{\text {máx }}^{*}}\right)$
$\min ($ Costo $)=\min \left\{\sum_{i}^{N_{C}} F_{i}\right\}$

donde $F_{i}=1$ si el $i$-esimo tubo que conecta dos módulos, de otra manera $F_{i}=0$. De este modo, la función de costo que se busca minimizar depende del número de caudalímetros a instalar.

Los problemas de optimización establecidos en las Ecs. (3) y (4) son problemas combinatorios del tipo $N P$ completo. Como herramienta de optimización se implementó el algoritmo de recocido simulado (Kirkpatrick et al., 1983). Este algoritmo está basado en una generalización de la estrategia del progreso iterativo, la cual comienza con una solución inicial y luego busca, dentro de su entorno, otra solución de menor costo. La generalización introducida por este método implica aceptar, con una probabilidad distinta de cero, una solución de mayor costo para evitar la permanencia en un mínimo local. Existen diferentes implementaciones de recocido simulado para problemas de múltiples objetivos (Suman y Kumar, 2006), en el presente trabajo se emplea el método propuesto por Suppapitnarm et al. (2000). 
Luego del proceso de sectorización se realizó el análisis de confiabilidad del sistema obtenido mediante el incremento de las demandas. Se perturbaron las demandas en un número fijo de nodos. Para una primera evaluación se decidió modificar un nodo por iteración, y para una etapa posterior se perturbaron diez nodos a la vez. El consumo de cada uno de los nodos se multiplicó por un factor que varía entre 1.1 y 5.6 con incrementos de 0.5. Para cada incremento se realizan 300 iteraciones escogiendo aleatoriamente los nodos cuyas demandas se modifican y se calculó, para cada caso, el valor de la resiliencia de red y el caudal aportado por la bomba. El valor de resiliencia de cada perturbación se promedia para las 300 iteraciones.

\section{Resultados}

De la primera etapa se tomó una solución que divide a la red en tres módulos, luego de la segmentación realizada por la segunda etapa se obtuvieron dos posibles configuraciones que se ilustran en la Figura 1. Es importante notar que para esta red la cantidad de comunidades en que puede ser dividida se ve limitada por la cantidad de fuentes, quedando definidas dos comunidades autónomas.

\section{SOLUCION 1}

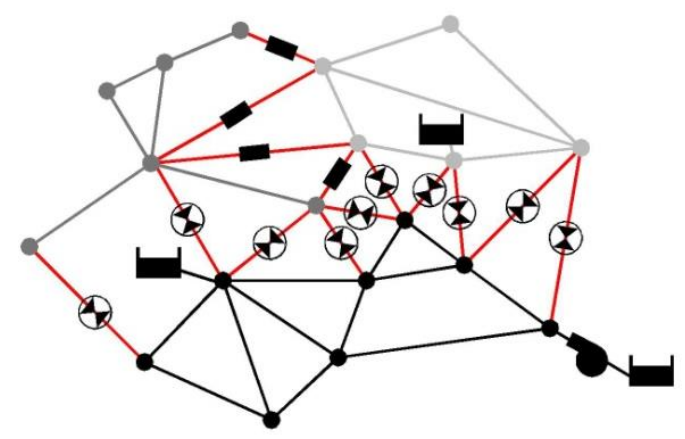

SOLUCION 2

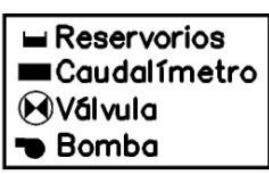

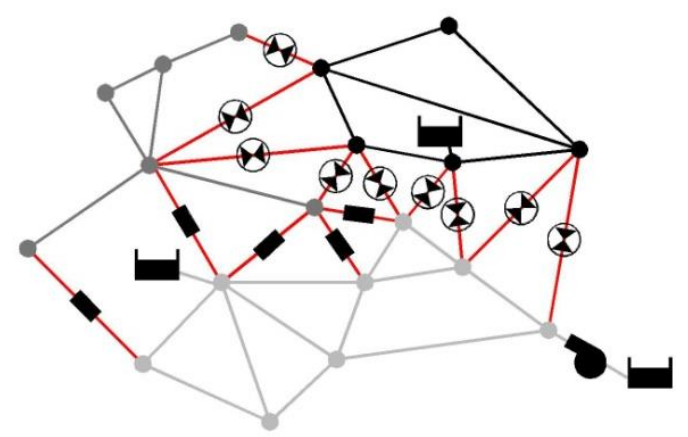

Figura 1: Soluciones obtenidas para la segmentación de la red Anytown.

Para las dos configuraciones que se muestran en la Figura 1, se perturbaron las demandas para comparar la confiabilidad de ambos sistemas. En las dos gráficas de la Figura 2 se muestran los valores de la resiliencia de cada solución y para cada esquema de perturbación, y en las dos gráficas de la Figura 3 se representan los caudales aportados por las bombas en galones por minuto. Se observa que cuando la demanda se incrementa en un solo nodo, el índice de resiliencia muestra un crecimiento sostenido, mientras que para incrementos en demandas de 10 nodos, la resiliencia muestra una etapa de aumento seguido por otra de disminución. Este hecho puede entenderse analizando los gráficos de los caudales aportados. Las gráficas de la figura 3 muestran que, a medida que se aumentan las demandas, las bombas impulsan caudales mayores para compensar las perturbaciones. En las gráficas podemos notar que cuando la bomba alcanza el límite de servicio, su caudal aportado comienza a disminuir impactando fuertemente en la resiliencia de la red.
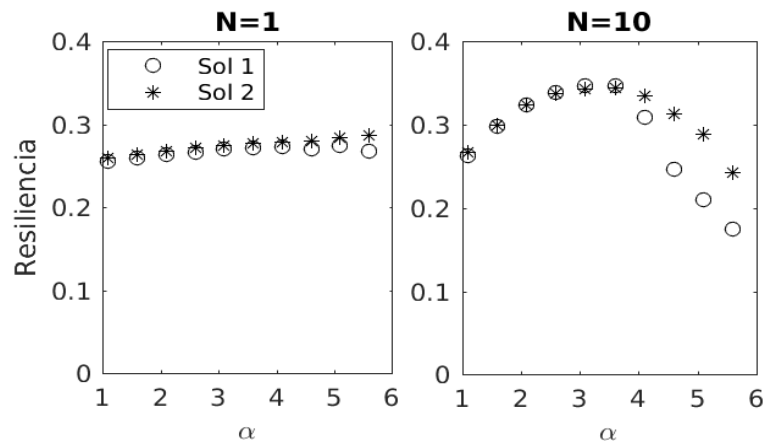

Figura 2: Resiliencia en función del parámetro de incremento en las demandas. Resultados para un nodo $(\mathrm{N}=1)$ y diez nodos $(\mathrm{N}=10)$ afectados. 


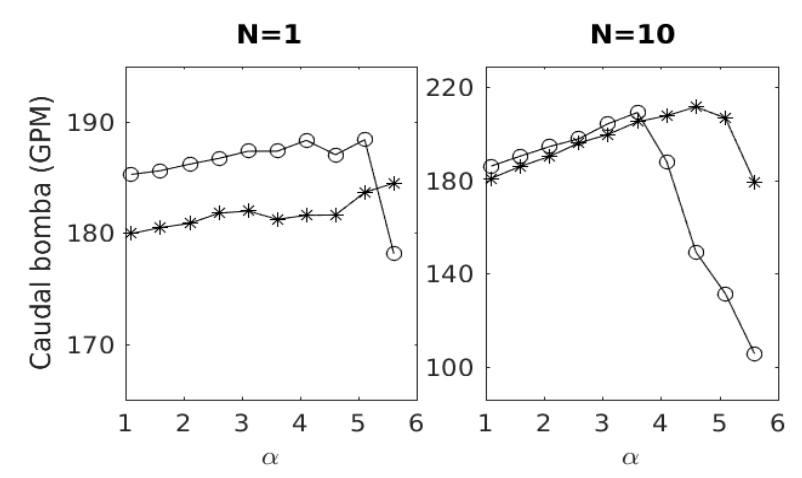

Figura 3: Resiliencia y caudal promedio aportado por la bomba en función del parámetro de incremento en las demandas.

Resultados para un nodo $(\mathrm{N}=1)$ y diez nodos $(\mathrm{N}=10)$ afectados.

Para un mayor análisis de los resultados, en la Figura 4 se presentan los diagramas de caja de los valores de resiliencia para las dos soluciones y cada esquema de perturbación. En las gráficas donde se presenta la perturbación de un nodo (izquierda) la resiliencia no presenta dispersión para incrementos de demanda $\alpha<3.1$, pero para incrementos mayores se observa que los valores de la resiliencia comienzan a dispersarse, teniendo mayor variabilidad para la solución 1 que para la solución 2. Esta tendencia se hace más notoria para los últimos valores de $\alpha$, donde la solución 1 comienza a tener valores inferiores atípicos hasta llegar al último escenario de perturbación donde la resiliencia toma valor cero.

Para las perturbaciones de diez nodos, se observa una situación similar. En la solución 1 (superior-derecha) comienzan a presentarse valores atípicos en el segundo escenario $(\alpha=1.6)$ y para el octavo y noveno $(\alpha=4.6, \alpha=$ 5.1) se observa que el valor de resiliencia decrece, y que más del $25 \%$ de los datos son iguales a cero, llegando en el último caso a tener una mediana igual a cero. Para la solución 2 (inferior-derecha) la perturbación de diez nodos afecta los valores de resiliencia, pero en contraposición con la solución 1, el decaimiento producido es menor. Esta solución muestra diagramas más homogéneos y menor cantidad de valores de resiliencia igual a cero.
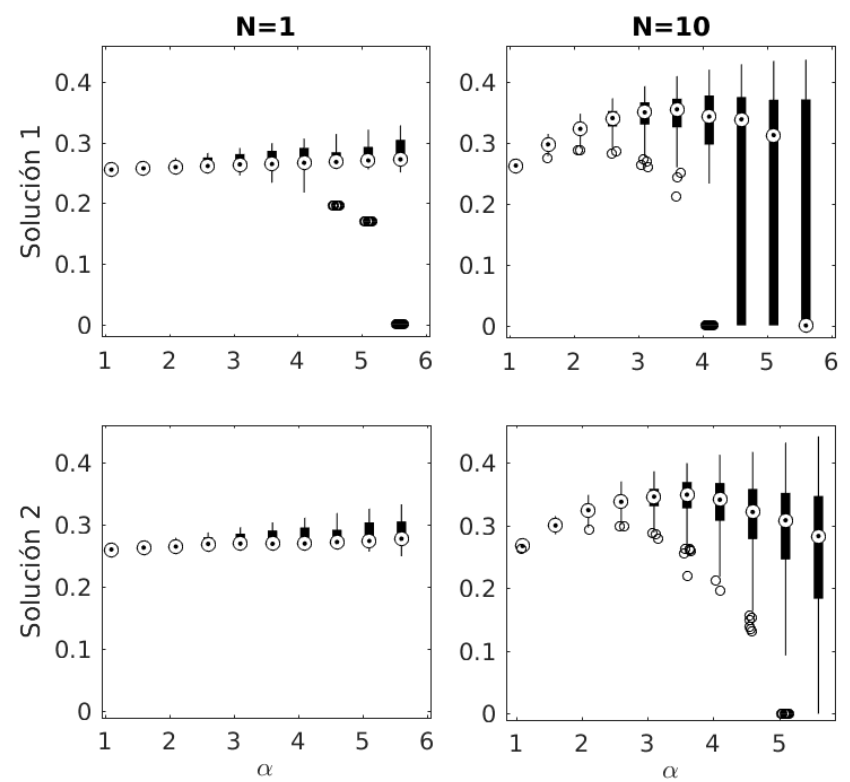

Figura 4: Valores de resiliencia de cada solución obtenidos para cada perturbación.

\section{Conclusiones}

En este trabajo se propuso una metodología para la sectorización de redes de agua, y se analizó la confiabilidad las soluciones óptimas encontradas. El enfoque desarrollado utiliza el concepto de resiliencia para evaluar el impacto que tiene los incrementos en las demandas en la red sectorizada. Se analizó el rendimiento hidráulico de las diferentes configuraciones de sectorización bajo dos diferentes esquemas de perturbación de las demandas. La metodología propuesta puede extenderse para analizar un número mayor de soluciones, ya que ofrece una 
herramienta selección entre un conjunto de soluciones óptimas de sectorización que está basado en la respuesta de la red frente a situaciones de aumento de las demandas.

\section{Referencias}

Alvisi S. y Franchini M. (2013). A heuristic procedure for the automatic creation of district metered areas in water distribution system. Urban Water Journal, 23.

Bianchotti J., Ercole P., Puccini, G. (2018). Detección de comunidades en redes de distribución de agua potable, Mecánica Computacional, 36, ISSN 2591-3522, 2059-2067.

Castro-Gamma M., Pan Q., Jonoski A., y Solomatine D. (2016). A graph theorical sectorization approach for energy reduction in water distribution networks. Proc. Engineering, 154:19-26.

Di Nardo A., Di Natale M., Santonastaso G., Tzatchkov V., y Yamanaka V.A. (2014). Divide and conquer partitioning techniques for smart water networks. Proc. Engineering, 89:1176-1183.

Herrera M., Abraham E., Wright R., Izquierdo J., y Pérez-García R. (2015). Medidas híbridas para el análisis de la resiliencia de redes de distribución de agua. Journal of Water Resources Planning and Management.

Kirkpatrick S., . J.G., y Vecchi M. (1983). Optimization by simulated annealing. Science, 220:671-680.

Newman M. y Girvan M. (2004). Finding and evaluating community structure in networks. Phys. Rev. E., 69(2).

Newman M. (2006). Modularity and community structure in netwoks. PNAS., 103(23).

Suman B. y Kumar P. (2006). A survey of simulated annealing as a tool for single and multiobjective optimization. Journal of the Operational Research Society, 57:1143-1160.

Suppapitnarm A., Seffen K., Parks G., y Clarkson P. (2000). Simulated annealing: analternative approach to true multiobjective optimization. Engineering Optimization, 33:59-85.

Todini E. (2000). Looped water distribution networks design using a resilience index based heuristic approach. Urban Water, 2:115-122. 